\title{
On the Relation between Tautly Imbedded Space Modulo an Analytic Subset $S$ and Hyperbolically Imbedded Space Modulo $S$
}

By

\author{
Yukinobu ADACHI*
}

\section{Introduction}

Let $X$ be a complex manifold, $M$ a relatively compact domain of $X$ and $S$ an analytic subset of $X$. We denote the open unit disk in the complex plane $C$ by $\Delta$, the polydisk $\Delta \times \cdots \times \Delta$ in $C^{k}$ by $\Delta^{k}$ and the Kobayashi pseudodistance of $M$ by $d_{M}$ (see [Ko] for its definition and basic properties). The space of holomorphic maps from a manifold $N$ to a manifold $M$ with compact-open topology will be denoted by $\operatorname{Hol}(N, M)$.

Following Kiernan-Kobayashi $[\mathrm{K}-\mathrm{K}]$ and $[\mathrm{L}]$, we use the following terminologies.

$M$ is tautly imbedded modulo $S$ in $X$ if for each positive integer $k$ and each sequence $\left\{f_{j}\right\}$ in $\operatorname{Hol}\left(\Delta^{k}, M\right)$ we have one of the following:

(a) $\left\{f_{j}\right\}$ has a subsequence which converges in $\operatorname{Hol}\left(\Delta^{k}, X\right)$;

(b) for each compact set $K \subset \Delta^{k}$ and each compact set $L \subset X \backslash S$, there exists an integer $N$ such that $f_{j}(K) \cap L=\phi$ for $j \geqq N$.

$M$ is hyperbolically imbedded modulo $S$ in $X$ if, for every pair of distinct points $p, q$ of $\bar{M}$, closure of $M$, not both contained in $S$, there exist neighborhoods $V_{p}$ and $V_{q}$ of $p$ and $q$ respectively in $X$ such that $d_{M}\left(V_{p} \cap M, V_{q} \cap M\right)>0$.

In [K-K], it was proved that if $M$ is tautly imbedded modulo $S$ in $X$, then $M$ is hyperbolically imbedded modulo $S$ in $X$ and brought up the inverse problem. But we believe there is no results except for the case $S=\phi$ (see Kiernan [Ki2] in case $S=\phi$ ). In this note, we deal with the inverse problem

Communicated by Y. Miyaoka, July 11, 1996.

1991 Mathematics Subject Classification(s): 32H02, 32H20

* 12-29 Kurakuen 2ban-cho, Nishinomiya-shi, Hyogo 662, Japan. 
for the case $S$ is a curve and $M$ and $X$ are special manifolds and give an affirmative answer (Theorem 4.4).

\section{$\S 1$ Degeneracy Locus of the Kobayashi Pseudodistance}

Throughout the sections $1 \sim 2$, let $X$ be a complex manifold, $M$ a relatively compact domain of $X$ and $d_{M}$ the Kobayashi pseudodistance of $M$. In [A-S2] we extended $d_{M}$ to $\bar{M}$, the closure of $M$ in $X$, as follows:

For $p, q \in \bar{M}$, we define

$$
\bar{d}_{M}(p, q)=\lim _{p^{\prime} \rightarrow p, q^{\prime} \rightarrow q} d_{M}\left(p^{\prime}, q^{\prime}\right), \quad p^{\prime}, q^{\prime} \in M .
$$

It is clear that $0 \leqq \bar{d}_{M}(p, q) \leqq \infty$. The function $\bar{d}_{M}$ does not satisfy the triangle inequality. For example, let $M=\{C \backslash\{0,1\}) \times \mathbb{C}, X=\mathbb{P}^{2}$, where $\mathbb{P}^{2}$ is the two-dimensional complex projective space, $p=[0: 0: 1], q=[1: 2: 1]$ and $r=[1: 3: 1]$. It is obvious that $\bar{d}_{M}(p, q)=\bar{d}_{M}(p, r)=0$. And $\bar{d}_{M}(q, r)=d_{M}(q, r)$ $\geqq d_{C \backslash\{0,1\}}(\pi(q), \pi(r))>0$, where $\pi$ is the projection of $M$ to $\mathbb{C} \backslash\{0,1\}$. So $\bar{d}_{M}$ is not a pseudodistance on $\bar{M}$.

Definition 1.1. We call $p \in \bar{M}$ a degeneracy point of $\bar{d}_{M}$ if there exists a point $q \in \bar{M} \backslash\{p\}$ such that $\bar{d}_{M}(p, q)=0$. By $S_{M}(X)$ we denote the set of the degeneracy points of $\bar{d}_{M}$ on $\bar{M}$ and call it the degeneracy locus of $\bar{d}_{M}$ in $X$.

Definition 1.2. (cf. [T] and [F]). A closed subset $E$ of $X$ will be called a pseudoconcave subset of order 1 , if for any coordinate neighborhood

$$
U:\left|z_{1}\right|<1, \cdots,\left|z_{n}\right|<1
$$

of $X$ and positive numbers $r, s$ with $0<r<1,0<s<1$ such that $U^{*} \cap E=\phi$, one obtains $U \cap E=\phi$, where

$$
U^{*}=\left\{p \in U ;\left|z_{1}(p)\right| \leqq r\right\} \cup\left\{p \in U ; s \leqq \max _{2 \leqq i \leqq n}\left|z_{i}(p)\right|\right\}
$$

In $[\mathrm{A}-\mathrm{S} 2]$, we proved the following

Theorem 1.3. The set $S_{M}(X)$ is a pseudoconcave subset of order 1 in $X$.

Let $S$ be an analytic subset of $X$. Using the extended function $\bar{d}_{M}$, we 
can show that $M$ is hyperbolically imbedded modulo $S$ in $X$ if and only if for every pair of distinct points $p, q \in \bar{M}$ not both contained in $S, \bar{d}_{M}(p, q)>0$ and $M$ is hyperbolically imbedded space in $X$ if and only if for every pair of distinct points $p, q \in \bar{M} \bar{d}_{M}(p, q)>0$, that is $S_{M}(X)=\phi$.

It is easy to see the following proposition.

Proposition 1.4. $M$ is hyperbolically imbedded modulo $S$ in $X$ if and only if $S \supset S_{M}(X)$.

\section{§2. Normality and Cluster Sets of a Sequence of Holomorphic Maps}

In [A-S1] we defined cluster sets of a sequence of holomorphic maps. Let a sequence $F=\left\{f_{j}\right\}$ in $\operatorname{Hol}\left(\Delta^{k}, M\right)$.

Definition 2.1. We define the cluster set $F(a: X)$ of $F$ at a point a of $\Delta^{k}$ by

$$
F(a: X)=\bigcap_{\varepsilon>0} \bigcap_{N=1}^{\infty} \bigcup_{j \geqq N} f_{j}\left(U_{\varepsilon}(a)\right)
$$

where $U_{\varepsilon}(a)=\left\{z \in \Delta^{k} ;\|z-a\|<\varepsilon\right\}$.

Let $F\left(\Delta^{k}: X\right)=\bigcup_{a \in \Delta^{k}} F(a: X)$.

Definition 2.2. A sequence $F=\left\{f_{j}\right\}$ in $\operatorname{Hol}\left(\Delta^{k}, M\right)$ is normal at $a \in \Delta^{k}$ if there exists a neighborhood $U$ of a such that every subsequence of $F$ has a convergent subsequence in $\operatorname{Hol}(U, X)$.

Clearly, we have

Proposition 2.3. If the cluster set $F(a: X)$ of a sequence $F$ in $\operatorname{Hol}\left(\Delta^{k}, M\right)$ consists of finite number of points of $X, F$ is normal at a.

Proposition 2.4. If there exist a point $a$ and a sequence of points $a_{j}$ in $\Delta^{k}$ such that $a_{j} \rightarrow a$ and $f_{j}\left(a_{j}\right) \rightarrow p \notin S_{M}(X)$, then $F$ is normal at $a$.

Proof. Since $S_{M}(X)$ is a closed set, there exists a closed neighborhood $V$ of $p$ biholomorphic to a closed unit ball in $X$ such that $V \cap S_{M}(X)=\phi$. If we define $\bar{d}_{M}(p, q)=\infty$ for $q \in X \backslash \bar{M}$, for some $\varepsilon>0, \bar{d}_{M}(p, \partial V) \geqq \varepsilon$ where $\partial V$ 
denotes the boundary of $V$ in $X$. Then $U(a)=\left\{z ; d_{\Delta^{k}}(a, z)<\frac{\varepsilon}{2}\right\}$ is relatively compact in $\Delta^{k}$. We shall prove $f_{j}(U(a)) \subset V$ for $j \geqq N$ where $N$ is a sufficiently large integer. If it is not true, we may assume $f_{j_{\lambda}}\left(b_{j_{\lambda}}\right) \in \partial V$ where $b_{j_{\lambda}} \in U(a)$, $b_{j_{\lambda}} \rightarrow b \in \overline{U(a)}$ and $f_{j_{\lambda}}\left(b_{j_{\lambda}}\right) \rightarrow q \in \partial V$ since $f_{j}\left(a_{j}\right) \in V$ for sufficiently large $j$. This is absurd, because

$$
\begin{aligned}
& \bar{d}_{M}(p, q) \leqq \lim _{j_{\lambda} \rightarrow \infty} d_{M}\left(f_{j_{\lambda}}\left(a_{j_{\lambda}}\right), f_{j_{\lambda}}\left(b_{j_{\lambda}}\right)\right) \\
& \leqq \lim _{j_{\lambda} \rightarrow \infty} d_{\Delta^{k}}\left(a_{j_{\lambda}}, b_{j_{\lambda}}\right)=d_{\Delta^{k}}(a, b) \leqq \frac{\varepsilon}{2}
\end{aligned}
$$

Corollary 2.5. (Theorem 1 in [Ki2]). If $S_{M}(X)=\phi$, then $F$ has a subsequence which converges in $\operatorname{Hol}\left(\Delta^{k}, X\right)$ and consequentry $M$ is tautly imbedded in $X$.

Corollary 2.6. If $F(a: X) \ni p$ and $p \notin S_{M}(X)$, then $F$ has a subsequence which converges in a neighborhood of $a$.

It is easy to see the following

Proposition 2.7. Let $S$ be a closed subset of $X$. Let $F$ be a sequence $\left\{f_{j}\right\}$ in $\operatorname{Hol}\left(\Delta^{k}, M\right)$. For each compact set $K$ and each compact set $L \subset X \backslash S$, there exists an integer $N$ such that $f_{j}(K) \cap L=\phi$ for $j \geqq N$ if and only if $F\left(\Delta^{k}: X\right) \subset S$.

\section{§3. An Auxiliary Theorem (Theorem 3.4)}

Lemma 3.1. Let $X$ be a complex manifold, $M$ be a relatively compact domain of $X$ and $F$ be a sequence $\left\{f_{j}\right\}$ in $\operatorname{Hol}\left(\Delta^{k}, M\right)$. Let $D$ be a convergence domain of $F$ with limit $f \in \operatorname{Hol}(D, X)$ and $D \cong \Delta^{k}$. If $a \in E=\partial D \backslash \partial \Delta^{k}$, then $F(a: X) \subset S_{M}(X)$.

Proof. We prove the lemma in 3 steps.

(1) We show if $F(a: X)=Q \cup S, Q \neq \phi, Q \cap S_{M}(X)=\phi$ and $S \subset S_{M}(X)$, then $Q=\{p\}$. If $Q \ni p_{1}, p_{2}$, there exist a neighborhood $U(a)$ and subsequences $F_{1}$, $F_{2}$ of $F$ such that $F_{i}$ converges to $f_{i}$ on $U(a)$ and $f_{i}(a)=p_{i}(i=1,2)$ from Corollary 2.6. Since $f_{1}$ and $f_{2}$ are analytic continuations of $f, p_{1}=p_{2}$ from the uniqueness of continuation.

(2) We show if $F(a: X)=\{p\} \cup S$ and $S \subset S_{M}(X)$ then $S=\phi$. Since $f$ has an 
analytic continuation at $a$ and $f(a)=p$ from Corollary 2.6, $f\left(a_{j}\right) \rightarrow p$ for every $a_{j} \in D$ such that $a_{j} \rightarrow a$. Let $\left\{a_{j}\right\}_{j=1,2, \ldots}$ be some points of $D$ such that $a_{j} \rightarrow a$ and $f\left(a_{j}\right)=p_{j}$ and $\varepsilon_{j}$ be positive numbers such that $\varepsilon_{j} \rightarrow 0$. There exists an integer $N_{1}$ such that $d\left(f_{j}\left(a_{1}\right), p_{1}\right)<\varepsilon_{1}$ for $j>N_{1}$, an integer $N_{2}$ such that $d\left(f_{j}\left(a_{1}\right), p_{1}\right)<\varepsilon_{2}$ and $d\left(f_{j}\left(a_{2}\right), p_{2}\right)<\varepsilon_{2}$ for $j>N_{2}$, an integer $N_{3}$ such that $d\left(f_{j}\left(a_{1}\right), p_{1}\right)<\varepsilon_{3}, d\left(f_{j}\left(a_{2}\right), p_{2}\right)<\varepsilon_{3}$ and $d\left(f_{j}\left(a_{3}\right), p_{3}\right)<\varepsilon_{3}$ for $j>N_{3}$ and so on, where $d$ is a distance on $X$. We may assume $N_{1}<N_{2}<N_{3}<\cdots$. Set $\tilde{a}_{j}=a_{1}$ for $j \leqq N_{2}, \tilde{a}_{j}=a_{2}$ for $N_{2}<j \leqq N_{3}, \tilde{a}_{j}=a_{3}$ for $N_{3}<j \leqq N_{4}$ and so on. Then $\tilde{a}_{j} \in D$, $\tilde{a}_{j} \rightarrow a$ and $f_{j}\left(\tilde{a}_{j}\right) \rightarrow p$. Let $q \in S$, then there exist $b_{\lambda} \in \Delta^{k}$ such that $b_{\lambda} \rightarrow a$ and $f_{j_{\lambda}}\left(b_{\lambda}\right) \rightarrow q$. Since there exists a sufficiently small closed neighborhood $V$ of $p$ such that $V \cap S_{M}(X)=\phi$, there exist $c_{\mu} \in \Delta^{k}$ such that $c_{\mu} \rightarrow a$ and $f_{j_{\lambda \mu}}\left(c_{\mu}\right) \rightarrow r \in \partial V$. This contradicts $Q=\{p\}$.

(3) If $F(a: X)=\{p\}, F$ is normal at $a$ from Proposition 2.3. Then $F$ converges on a neighborhood of $a$ from Vitali's theorem. This is absurd. If $Q=\phi$, there is no problem.

Lemma 3.2. Let $A$ and $S$ be curves of $\boldsymbol{P}^{2}$ and set $X=\boldsymbol{P}^{2}$ and $M=\boldsymbol{P}^{2} \backslash A$. Let $M$ be tautly imbedded modulo $S$ in $X$ and let $F$ be a sequence $\left\{f_{j}\right\}$ in $\operatorname{Hol}\left(\Delta^{k}, M\right)$ without any convergent subsequence in $\operatorname{Hol}\left(\Delta^{k}, X\right)$. Let $D \neq \phi$ be the convergence domain of $F$ with limit $f \in \operatorname{Hol}(D, X)$. Set $E=\Delta^{k} \backslash D$. Then either $E$ is contained in an analytic subset of $\Delta^{k}$ or $f(D) \subset S_{M}(X)$.

Proof. Since $M$ is hyperbolically imbedded modulo $S$ in $X$ from Theorem 1 in $[\mathrm{K}-\mathrm{K}], S \supset S_{M}(X)$ from Proposition 1.4. Then $S_{M}(X)=\phi$ or a curve from Theorem 1.3. Since $F$ has not any convergent subsequence in $\operatorname{Hol}\left(\Delta^{k}, X\right), S_{M}(X)$ is a curve from Corollary 2.5. Since $F\left(\Delta^{k}: X\right) \subset S$ from Proposition 2.7, $f(D) \subset S$. Suppose $f(D) \subset S_{0}$ and $f(D) \not \subset S_{M}(X)$ where $S_{0}$ is an irreducible component of $S$ which is not contained in $S_{M}(X)$. If $f\left(a_{j}\right) \rightarrow p$ for a point $a \in \partial E \backslash \partial \Delta^{k}$, a sequence of points $a_{j} \in D$ and $a_{j} \rightarrow a, p \in S_{0} \cap S_{M}(X)$ from Lemma 3.1. There is a rational function $g$ on $\boldsymbol{P}^{2}$ which takes zero only on $S_{M}(X)$ and takes pole only on a line $L$ such that $L \cap S_{M}(X) \cap S_{0}=\phi$. Then the points of indeterminancy of $g$ are contained in $\boldsymbol{P}^{2} \backslash S_{0}$. So $\Phi=g \circ f$ is meromorphic in $D$ and has not a point of indeterminancy. And $\lim _{z \rightarrow a} \Phi(z)=0$ for $z \in D$ and $a \in \partial E \backslash \partial \Delta^{k}$. Therefore $\bar{P} \cap\left(\partial E \backslash \partial \Delta^{k}\right)=\phi$ where $P$ denotes the pole divisor of $\Phi$.

If we set $\Phi \equiv 0$ on $E$, then $\Phi$ is continuous on $Y=\Delta^{k} \backslash P, E$ is contained in the zeros of $\Phi$ and $\Phi$ is holomorphic in $Y \backslash E$. So $\Phi$ is holomorphic in $Y$ from Rado's 
theorem. Therefore either $\Phi \equiv 0$ on $\Delta^{k}$ or $\Phi \not \equiv 0$ and $E$ is contained in an analytic subset of $\Delta^{k}$. The former case contradicts to the assumption since $f(D) \not \subset S_{M}(X)$.

Lemma 3.3. Let $A_{1}, \cdots, A_{l}$ be $l(l \geqq 1)$ irreducible hypersurfaces of $\mathbb{P}^{n}$ and set $X=\mathbb{P}^{n}, M=\mathbb{P}^{n} \backslash\left(A_{1} \cup \cdots \cup A_{l}\right)$. Let $\left\{f_{j}\right\}$ be in $\operatorname{Hol}\left(\Delta^{k}, M\right), E$ be an analytic subset of $\Delta^{k}$ and $\left\{f_{j}\right\}$ converge to $f$ in $\operatorname{Hol}\left(\Delta^{k} \backslash E, X\right)$. Then either $\left\{f_{j}\right\}$ converges to $f$ in $\operatorname{Hol}\left(\Delta^{k}, X\right)$ or $f\left(\Delta^{k} \backslash E\right) \subset \bigcap_{i=1}^{l} A_{i}$.

Proof. Since $f_{j}\left(\Delta^{k}\right) \cap A_{i}=\phi$ for all $j$, we have, from Hurwitz's theorem, either $f\left(\Delta^{k} \backslash E\right) \cap A_{i}=\phi$ or $f\left(\Delta^{k} \backslash E\right) \subset A_{i}$ for each $i=1, \cdots, l$. Therefore, if $f\left(\Delta^{k} \backslash E\right) \not \subset \bigcap_{i=1}^{l} A_{i}$, then $f\left(\Delta^{k} \backslash E\right) \cap A_{i}=\phi$ for some $i$. Since $\mathbb{P}^{n} \backslash A_{i}$ is a Stein manifold, it is imbedded into $\mathbb{C}^{N}$ by $\Phi$. Recalling the maximum principle, $\Phi \circ f_{j}$ converges in $\operatorname{Hol}\left(\Delta^{k}, C^{N}\right)$. Therefore $\left\{f_{j}\right\}$ converges in $\operatorname{Hol}\left(\Delta^{k}, \mathbb{P}^{n} \backslash A_{i}\right)$.

Theorem 3.4. Let $A$ be a curve of $\boldsymbol{P}^{2}$ whose number of irreducible components is greater than 1 and $S$ be a curve of $\mathbb{P}^{2}$. Set $X=\mathbb{P}^{2}$ and $M=\mathbb{P}^{2} \backslash A$. If $M$ is tautly imbedded modulo $S$ in $X$, then $M$ is tautly imbedded modulo $S_{M}(X)$ in $X$.

Proof. Since $S \supset S_{M}(X), S_{M}(X)=\phi$ or a curve. If $S_{M}(X)=\phi$, above theorem is correct from Corollary 2.5. So we assume $S_{M}(X)$ is a curve and show that $F\left(\Delta^{k}: X\right) \subset S_{M}(X)$ if $F$ be a sequence $\left\{f_{j}\right\}$ in $\operatorname{Hol}\left(\Delta^{k}, M\right)$ which has not any convergent subsequence in $\operatorname{Hol}\left(\Delta^{k}, X\right)$.

Suppose there exists a point $a \in \Delta^{k}$ such that $F(a: X) \ni p \notin S_{M}(X)$. Then there are a subsequence $F^{\prime}$ of $F$ and a neighborhood $U(a)$ of $a$ such that $F^{\prime}$ converges to $f$ in $\operatorname{Hol}(U(a), X)$ from Corollary 2.6. Let $D$ be a convergence domain of $F^{\prime}$ which contains $U(a)$. From the assumption $D \subsetneq \Delta^{k}$. Set $E=\Delta^{k} \backslash D$. From Lemma $3.2 E$ is contained in an analytic subset of $\Delta^{k}$. From Lemma $3.3 f\left(\Delta^{k} \backslash E\right) \subset \bigcap_{i=1}^{l} A_{i}$, where $A_{1}, \cdots, A_{l}$ are irreducible components of A. If $\bigcap_{i=1}^{l} A_{i}=\phi$, it is a contradiction since $f\left(\Delta^{k} \backslash E\right) \neq \phi$. If $\bigcap_{i=1}^{l} A_{i}=\left\{q_{1}\right\}$ $\cup \cdots \cup\left\{q_{t}\right\}, f\left(\Delta^{k} \backslash E\right)=\left\{q_{s}\right\}=\{p\} \quad(1 \leqq s \leqq t)$. So $F^{\prime}(E: X) \ni p$. This is a contradiction since $F^{\prime}(E: X) \subset S_{M}(X)$ from Lemma 3.1. 
Corollary 3.5. Let $A$ be a curve of $\mathbb{P}^{2}$ whose number of irreducible components is greater than 1. Set $X=\boldsymbol{P}^{2}$ and $M=\boldsymbol{P}^{2} \backslash A$. If $S_{M}(X) \subset A, \boldsymbol{P}^{2} \backslash A$ is tautly imbedded modulo $S_{M}(x)$ in $X$.

Proof. Since $\boldsymbol{P}^{2} \backslash A$ is hyperbolically imbedded modulo $S_{M}(X)$ in $\boldsymbol{P}^{2}$ and $S_{M}(X) \subset A, P^{2} \backslash A$ is complete hyperbolic from Theorem 4 in $[\mathrm{K}-\mathrm{K}]$. Then $\boldsymbol{P}^{2} \backslash A$ is taut from [E] and [Ki1]. By the definition, $\boldsymbol{P}^{2} \backslash A$ is tautly imbedded modulo $A$ in $\boldsymbol{P}^{2}$. So $\boldsymbol{P}^{2} \backslash A$ is tautly imbedded modulo $S_{M}(X)$ in $\boldsymbol{P}^{2}$ by Theorem 3.4 .

\section{§4. Theorem 4.4}

In $[\mathrm{A}-\mathrm{S} 1]$ we defined a nonhyperbolic curve as follows.

Definition 4.1. Let $A$ be curve of $\boldsymbol{P}^{2}$. An irreducible curve $C$ of $\boldsymbol{P}^{2}$ will be called a nonhyperbolic curve with respect to $A$ if the normalization of $C \backslash A$ is isomorphic to $C$ or $C^{*}=C \backslash\{0\}$. If $C$ is an irreducible component of $A$, we shall say that $C$ is a nonhyperbolic curve with respect to $A$ if the normalization of $C \backslash A^{\prime}$ is isomorphic to $C, C^{*}, P^{1}$ or an elliptic curve, where $A^{\prime}$ is the union of the components of $A$ except $C$ which may be $\phi$.

Theorem 4.2. (Theorem 2 in [A-S1]). Let $A$ be a curve with $l(l \geqq 4)$ irreducible components of $\boldsymbol{P}^{2}$. Set $X=\boldsymbol{P}^{2}$ and $M=\boldsymbol{P}^{2} \backslash A$. Suppose that the number of the nonhyperbolic curves of $\boldsymbol{P}^{2}$ with respect to $A$ is finite, then there is a curve $S$ of $\boldsymbol{P}^{2}$ such that $M$ is tautly imbedded modulo $S$ in $X$. Here we may take $S=\phi$ if there is no nonhyperbolic curve of $\boldsymbol{P}^{2}$ with respect to $A$.

Theorem 4.3. (Corollary of Theorem in [A]). Let $A$ be a curve with $l$ $(l \geqq 4)$ irreducible components of $\boldsymbol{P}^{2}$. Set $X=\boldsymbol{P}^{2}$ and $M=\boldsymbol{P}^{2} \backslash A$.

(1) If the number of the nonhyperbolic curves of $\boldsymbol{P}^{2}$ with respect to $A$ is at most finite, $S_{M}(X)$ is empty or a curve.

(2) If the number of the nonhyperbolic curves of $\boldsymbol{P}^{2}$ with respect to $A$ is infinite, then $S_{M}(X)=X$.

Therefore, if $S_{M}(X)$ is a curve, there is a curve $S$ of $P^{2}$ such that $M$ is tautly imbedded modulo $S$ in $X$ by Theorem 4.3 and Theorem 4.2. And then, $M$ is tautly imbedded modulo $S_{M}(X)$ in $X$ from Theorem 3.4. So we have the following 
Theorem 4.4. Let $A$ be a curve with $l(l \geqq 4)$ irreducible components of $\boldsymbol{P}^{2}$. Set $X=\boldsymbol{P}^{2}$ and $M=\boldsymbol{P}^{2} \backslash A$. If $S_{M}(X)$ is a curve, $M$ is tautly imbedded modulo $S_{M}(X)$ in $X$.

Remark. Let $X$ and $M$ be the same in Theorem 4.4 and $S$ be a curve of $X$. If $M$ is hyperbolically imbedded modulo $S$ in $X, M$ is tautly imbedded modulo $S$ in $X$. Because, $S_{M}(X) \subset S$ by Proposition 1.4 and $S_{M}(X)$ is a curve or an empty set by Theorem 1.3. So $M$ is tautly imbedded modulo $S_{M}(X)$ in $X$ by Theorem 4.4 and Corollary 2.5. Therefore $M$ is tautly imbedded modulo $S$ in $X$.

\section{References}

[A] Adachi, Y., On the hyperbolicity of projective plane with lacunary curves, J. Math. Soc. Japan, 46 (1994), 185-193.

[A-S1] Adachi, Y. and Suzuki, M., On the family of holomorphic mappings into projective space with lacunary hypersurfaces, J. Math. Kyoto Univ., 30 (1990), 451-458.

[A-S2] - Degeneracy points of the Kobayashi pseudodistances on complex manifolds, Proc. Symp. Pure Math. 52 (1991), Several Complex Variables and Complex Geometry, Part 2, 41-51.

[E] Eisenman, D., Holomorphic mappings into tight manifolds, Bull. Amer. Math. Soc., 76 (1970), 46-48.

[F] Fujita, O., Sur les familles d'ensembles analytiques, J. Math. Soc. Japan, 16 (1964), 379-405.

[Ki1] Kiernan, P., On the relations between taut, tight and hyperbolic manifolds, Bull. Amer. Math. Soc., 76 (1970), 49-51.

[Ki2] - Hyperbolically imbedded spaces and the big Picard theorem, Math. Ann., 204 (1973), 203-209.

[K-K] Kiernan, P. and Kobayashi, S., Holomorphic mappings into projective space with lacunary hyperplanes, Nagoya Math. J., 50 (1973), 199-216.

[Ko] Kobayashi, S., Hyperbolic manifolds and holomorphic mappings, Marcel Dekker, New York, 1970.

[L] Lang, S., Introduction to complex hyperbolic spaces, Springer-Verlag, 1987.

[T] Tadokoro, M., Sur les ensembles pseudoconcaves généraux, J. Math. Soc. Japan, 17 (1965), 281-290. 